\title{
Adaptive Voltage Control of Distribution Network with High Proportion PV
}

\author{
Junchao $\mathrm{Ma}^{1}$, Xiaoming Huang ${ }^{1}$, Boliang Lou ${ }^{1}$, Xiulin $\mathrm{Xiao}^{2}$, Yinlong Fan ${ }^{2}$ and Dan Zhou ${ }^{3 *}$ \\ ${ }^{1}$ State Grid Zhejiang Electric Power Co., Ltd. Research Institute, Hangzhou, Zhejiang, 310014, China \\ ${ }^{2}$ Hangzhou Yineng Power Technology Co., Ltd., Hangzhou, Zhejiang, 310014, China \\ ${ }^{3}$ College of Information Engineering, Zhejiang University of Technology, Hangzhou, Zhejiang, 310014, China
}

\begin{abstract}
With the increase of grid-connected PV capacity, voltage regulation at point of common coupling by controlling the reactive power injected into the grid is available. This paper presents an adaptive voltage control strategy for distribution network with high proportion PV system. The PI gain of the voltage controller is automatically adjusted by the extremum seeking algorithm to dynamically respond to the changes of the network. The PI gains are updated online through the minimization of a cost function, which represents the voltage controller performance. Finally, a distribution network model of $5 \mathrm{MW}$ photovoltaic power generation system is built in MATLAB / Simulink to verify the effectiveness of the proposed control strategy.
\end{abstract}

\section{Introduction}

In recent years, with the decrease of photovoltaic system cost and the improvement of photovoltaic cell efficiency, more and more photovoltaic systems are connected to the distribution network, forming a distribution network with high proportion of photovoltaic system.

With the increase of photovoltaic grid-connected capacity, the photovoltaic system can provide auxiliary services of voltage regulation by controlling the power electronic converter [1]. Photovoltaic systems are mainly connected to the grid through voltage source converters (VSC). Because the photovoltaic system dynamically adjusts the voltage at the grid connected node through voltage source converter (VSC). Therefore, photovoltaic system can act as a good voltage regulator in the distribution network $[2,3]$.

The traditional PI controller has been widely used in VSC control in the distribution network. However, distributed energy generation has great randomness, and it can not accurately follow the change of power when using fixed PI controller. Therefore, the fixed gain PI controller is not suitable for dynamic distributed energy generation [4]. In addition, the traditional PI controller parameter adjustment depends on engineering experience. However, in the power system, the structure of the power system is complex and changes rapidly, so it is difficult to adapt to the changes of the system by manual analysis [5].
Therefore, in order to overcome the shortcomings of traditional PI control, an adaptive voltage control method based on extremum search for distribution network with high proportion of photovoltaic system is proposed. The PI parameters of the voltage controller are updated online to dynamically respond to system changes by minimizing the cost function representing the performance of the voltage controller. The simulation model of 5MW photovoltaic grid connected power generation system is established in Matlab/Simulink, and the proposed adaptive voltage controller is tested. The proposed control method does not need system model or parameters, and is easy to implement. Compared with the traditional PI controller, the proposed control method can better adapt to load changes and grid faults, which can improve the reliability and voltage stability of power system.

\section{Voltage control of common connection point}

\subsection{Control of grid connected VSC}

The proposed control scheme of three-phase grid connected VSC is based on instantaneous power theory of synchronous rotating $d q$ coordinate system, as shown in figure 1. The control system consists of five parts: MPPT controller, PCC voltage controller, vdc regulator, current controller and PWM generator. 


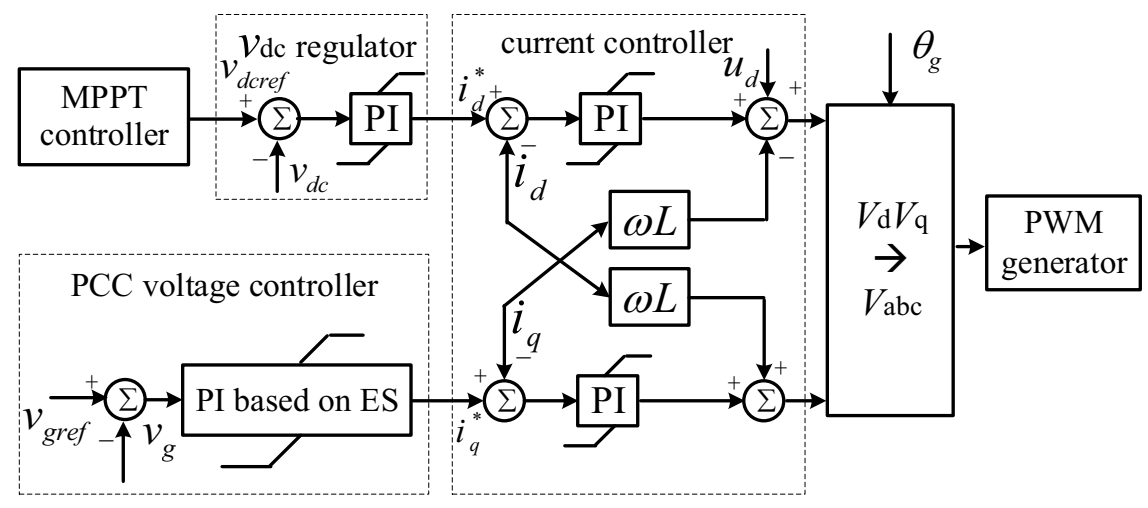

Figure 1. The proposed control scheme for the grid-connected VSC

\subsection{PCC voltage control}

Figure 1 shows the control block diagram of using PI controller to adjust PCC voltage. The PCC voltage is measured and its RMS value $v g$ is calculated, and then $v g$ is compared with the reference voltage vgref. The error between the actual voltage and the reference voltage is input into the voltage controller based on extremum search to adjust the reference output current component $i q$, that is, $v g$ is adjusted to the reference value of reactive current of vgref. The control mode can be specifically expressed as follows:

$$
i_{q}^{*}=K_{P}\left[v_{\text {gref }}(t)-v_{g}(t)\right]+K_{I} \int_{0}^{t}\left[v_{\text {gref }}(t)-v_{g}(t)\right] d t
$$

where $K P$ and $K I$ are the proportional and integral gain parameters of PI controller. The two gain parameters of PI controller affect the dynamic performance of voltage regulation. Therefore, setting gain to optimize the performance of PI controller is an important issue for effective and efficient voltage regulation. In this paper, an extremum search algorithm without model is used to automatically adjust the gain parameters of PI online.

\section{Gain self-tuning of PI controller based on extremum search}

In this section, two extremum search control algorithms based on scalar gradient are applied, which are ES1 for time independent cost function (static optimization) and ES2 for time-varying cost function (function optimization). According to the different cost functions selected, two extremum search algorithms are used to design the gain self-tuning method of PI controller.

\subsection{ES1 for static optimization}

Figure 2 shows the basic framework of a controller based on gradient extremum search algorithm. The algorithm injects a sinusoidal vibration quantity asin $\omega t$ into the system to output a cost function $Q(\hat{\theta})$.

The cost function is then multiplied by asin $\omega t$, and the resulting signal $\dot{\xi}$ is an estimate of the gradient change of the cost function relative to $\theta$. The estimated value is added to the modulated signal asin $\omega$ t after passing the integrator $\mathrm{kai} / \mathrm{s}$. The corresponding equations of the basic multi parameter extremum search algorithm are as follows:

$$
\begin{gathered}
\dot{\xi}=a_{i} k_{a i} \sin \left(\omega_{i} t\right) Q(\hat{\theta}) \\
\hat{\theta}_{i}=\xi_{i}+a_{i} \sin \left(\omega_{i} t\right)
\end{gathered}
$$

where ai is the gain, $\omega i$ is the frequency, $i$ is an integer, $\omega i>\omega^{*}$ and $\omega^{*}$ is large enough to ensure convergence. If the parameters $a i, \omega i$ and $k a i$ are properly selected, the output cost function $Q(\hat{\theta})$ will converge to the optimal cost function value $Q\left(\theta^{*}\right)$.

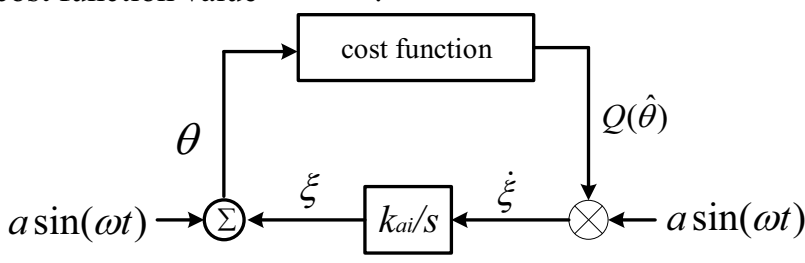

Figure 2. Block diagram of the basic gradient-based extremum seeking control algorithm

\subsection{ES2 for function optimization}

Figure 3 shows a block diagram of an extreme value search algorithm for function optimization. There is no disturbance signal between the integral function and the system, which is different from the basic extremum search (such as ES1) algorithm. The corresponding multi parameter extremum search algorithm equation is as follows:

$$
\dot{\hat{\theta}}_{i}=a \sqrt{\omega_{i}} \cos \left(\omega_{i} t\right)-k \sqrt{\omega_{i}} \sin \left(\omega_{i} t\right) Q(\hat{\theta}, t)
$$

where a and $\mathrm{k}$ are positive numbers, $\omega_{i}>\omega^{*}$ and $\omega^{*}$ are large enough to ensure convergence. 


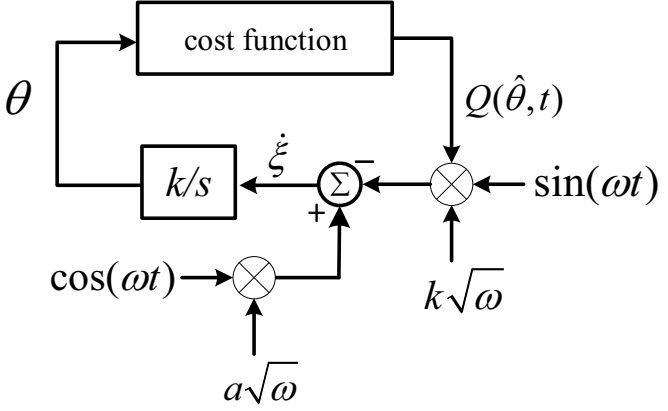

Figure 3. Block diagram of the extremum seeking control algorithm for the case of functional optimization

\subsection{PI controller gain self-tuning}

The PI parameters of PCC voltage controller are adjusted by extremum search algorithm to minimize the tracking error of the closed-loop system with unknown objects. The control effect of PI controller is quantified by cost function. In this paper, two cost functions are designed for ES1 and ES2 respectively.

For ES1, the voltage deviation from the beginning of voltage change (e.g. voltage change caused by load change) to the end of voltage change is integrated, and this integral value is taken as the cost function.

$$
Q(\theta, t)=\int_{t_{0}}^{T}\left[v_{\text {gref }}(t)-v_{g}(t)\right]^{2} d t
$$

where $t 0$ and $T$ are the starting and ending times of voltage change when calculating the cost function, and $\theta$ is the vector containing a set of PI parameters. That is $\theta=\left[K_{P}, K_{I}\right]^{T}$.

For ES1, the PI gain value is updated with equation (6):

$$
\left\{\begin{array}{l}
\dot{\xi}_{K p}=a_{K p} \sin \left(\omega_{1} t\right) Q\left(\hat{\theta}_{K p}, t\right) \\
\hat{K}_{p}(t)=\xi_{K p}(t)+a_{K p} \sin \left(\omega_{1} t\right) \\
\dot{\xi}_{K_{I}}=a_{K_{I}} \sin \left(\omega_{2} t\right) Q\left(\hat{\theta}_{K_{I}}, t\right) \\
\hat{K}_{I}(t)=\xi_{K_{I}}(t)+a_{K_{I}} \sin \left(\omega_{2} t\right)
\end{array}\right.
$$

The extremum search algorithm uses the discrete values of the cost function generated at the end of each iteration to calculate the next set of controller parameters $\theta$. When a new voltage change occurs, the PI controller will use the new controller parameters to execute and continue in this iterative manner until the optimal parameters are found.

For ES2, the following cost function calculated at each sampling time $\Delta \mathrm{t}$ is used:

$$
Q(\theta, \Delta t)=\left[v_{\text {gref }}(t)-v_{g}(t)\right]^{2}
$$

For ES2, the PI gain value is updated with equation (8):

$$
\begin{aligned}
& \dot{\hat{K}}_{P}(t)=a_{1} \sqrt{\omega_{1}} \cos \left(\omega_{1} t\right)-k_{1} \sqrt{\omega_{1}} \sin \left(\omega_{1} t\right) Q\left(\hat{\theta}_{K_{P}}, \Delta t\right) \\
& \dot{\hat{K}}_{I}(t)=a_{2} \sqrt{\omega_{2}} \cos \left(\omega_{2} t\right)-k_{2} \sqrt{\omega_{2}} \sin \left(\omega_{2} t\right) Q\left(\hat{\theta}_{K_{I}}, \Delta t\right)
\end{aligned}
$$

Similarly, the extremum search algorithm uses the discrete value of the cost function generated at each sampling time to calculate the PI controller parameter $\theta$ at the next sampling time. The block diagram of PI global parameter searching is shown in figure 4 .

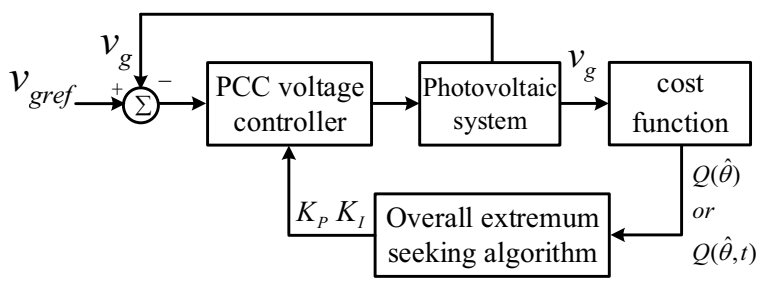

Figure 4. Overall extremum seeking PI tuning scheme

\section{Simulation analysis}

In this section, the proposed adaptive voltage controller based on extremum search is tested in the distribution network model. The PV array consists of 1720 parallel battery strings, each of which contains seven modules with a maximum output power of $415 \mathrm{~W}$, forming a $5 \mathrm{MW}$ photovoltaic system. The power electronics interface consists of a DC / AC converter and two DC side capacitors with a voltage of $520 \mathrm{~V}$. The converter model adopts IGBT bridge three-level VSC based on PWM control. The inverter choke RL and a filter capacitor are used to filter the harmonic generated by IGBT bridge. A $250 \mathrm{~V} / 25 \mathrm{kV}$ three-phase transformer is used to connect the inverter to the distribution network. The distribution network consists of a $25 \mathrm{kV}$ feeder, a load and an equivalent $120 \mathrm{kv}$ transmission system. PCC voltage is represented by $v g$, which can control inverter to adjust PCC voltage by generating or consuming a certain amount of reactive power.

The voltage controller based on ES1 is tested in the case of load change, and the performance of voltage controller based on ES2 algorithm is tested in case of power grid fault. The performance of the voltage controller in the two cases is compared with that of the traditional PI controller. The control parameters for simulation are listed in Table 1.

Table 1. Control Parameters of ES algorithms

\begin{tabular}{llllll}
\hline \multirow{2}{*}{$\mathrm{ES} 1$} & $a_{1}$ & $\omega_{1}$ & $a_{2}$ & $\omega_{2}$ & $k_{a i}$ \\
\cline { 2 - 6 } & 1 & $\mathrm{pi} / 100$ & 10 & $0.15 \mathrm{pi}$ & 1 \\
\hline \multirow{2}{*}{$\mathrm{ES} 2$} & $a_{1}$ & $\omega_{1}$ & $a_{2}$ & $\omega_{2}$ & $k_{1}, k_{2}$ \\
\cline { 2 - 6 } & 1 & $\mathrm{pi} / 100$ & 25 & $0.06 \mathrm{pi}$ & 1 \\
\hline
\end{tabular}

\section{1 comparison of the effect of voltage controller when load changes}

If there is load change in the system, the voltage at PCC of distribution network will change. Therefore, a voltage controller is required to maintain the voltage within a specific range of normal voltage, especially when the proportion of renewable energy increases. Figure 5 shows the simulation results of the system with or without voltage controller when $3.75 \mathrm{MW}$ reactive load is switched on and off at PCC. Obviously, if there is no voltage controller to inject reactive power into the grid, the voltage drops to $0.96 \mathrm{pu}$ due to load changes. On the contrary, 
when the voltage controller is used, the voltage returns to normal. Therefore, the performance of the voltage

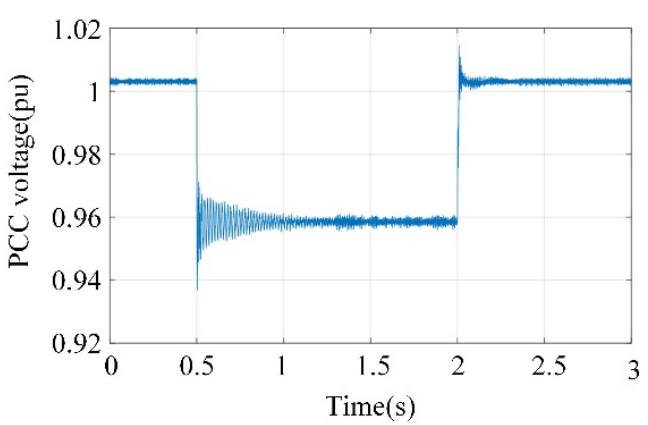

(a) with voltage controller controller is very important to the system, and its parameters need to be adjusted adaptively online.

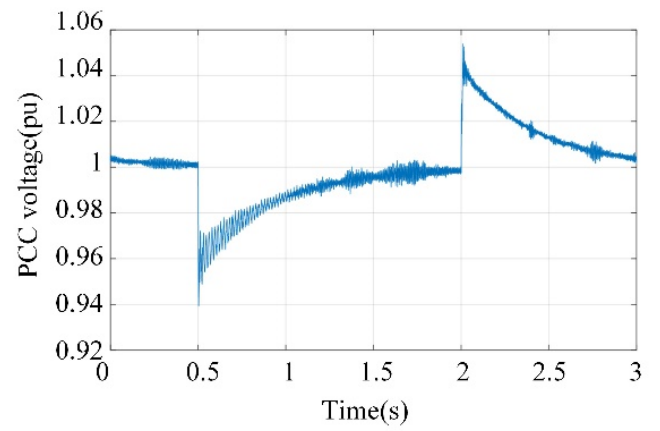

(b) without voltage controller

Figure 5. PCC voltage under load variation with and without voltage controller

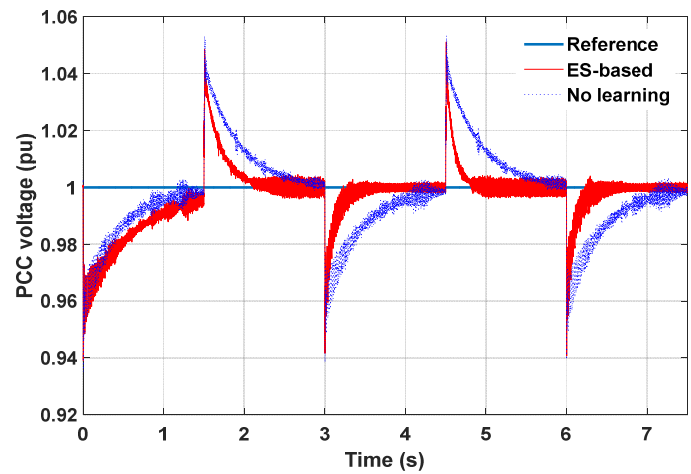

Figure 6. PCC voltage under load variations with voltage controller based on ES1

\subsection{PCC voltage based on Es1 voltage controller}

The PI gain of voltage controller is adjusted adaptively by ES1 algorithm to obtain better voltage control performance of PCC. In order to verify the effectiveness of the proposed adaptive parameter adjustment algorithm, the same $3.75 \mathrm{MW}$ load is used and the switch is conducted every $1.5 \mathrm{~s}$. The simulation results are shown in figure 6 . It takes about 1.5 seconds for the traditional PI controller to restore the voltage to the normal value. However, after learning for a certain period of time, the PCC voltage returned to the normal value within 0.5 seconds after using the ES1 based method. Figure 7 shows the change of reactive power during load change. The controller injects a certain amount of reactive power into the grid to maintain PCC voltage. Using adaptive voltage controller, reactive power injection is faster than traditional PI controller.

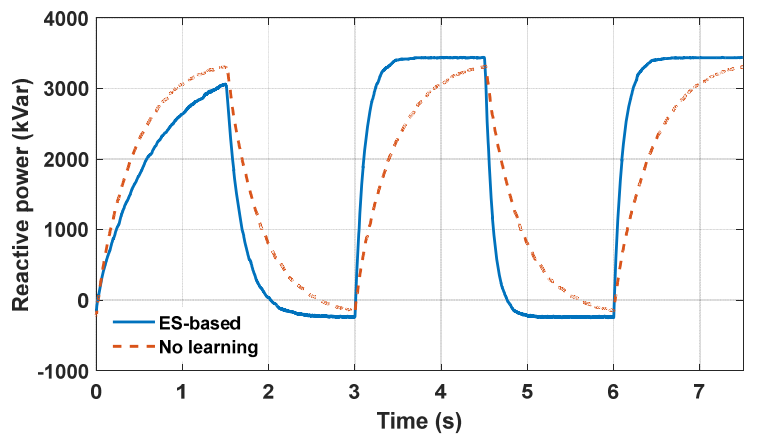

Figure 7. Reactive power variations by the voltage controller during load changes

\section{Conclusion}

This paper presents an adaptive voltage control method for distribution network with high proportion photovoltaic system. The PI gain of voltage controller is adjusted adaptively by extremum search algorithm to dynamically respond to system changes. The PI gain of the voltage controller is updated online by minimizing the cost function reflecting the performance of the voltage controller. A 5MW photovoltaic system is constructed by Matlab/Simulink, and the adaptive voltage controller based on extremum search is tested on the distribution network model. Simulation results show that, compared with the traditional PI controller, the proposed voltage controller can easily adapt to load changes and grid faults, thus improving the reliability and voltage stability of 
power system.

\section{Acknowledgments}

The work was supported in part by Key Research and Development Program of Zhejiang Province under Grant 2019C01149, in part by State Grid Corporation Science and Technology Project under Grant 5211DS180031, in part by National Natural Science Foundation of China under Grant 61903333, in part by Zhejiang Provincial Natural Science Foundation of China under Grant LQ19F030008

\section{References}

1. Zeraati, M., Golshan, M., and Guerrero, J. (2019) Voltage quality improvement in low voltage distribution networks using reactive power capability of single-phase PV inverters. IEEE Trans. Smart Grid, in Press, 10:5057-5065.

2. Li, H., Li, F., Xu, Y. (2010) Adaptive voltage control with distributed energy resources: algorithm, theoretical analysis, simulation, and field test verification. IEEE Trans. Power Syst., 25:1638-1647.

3. Wang, T., O'Neill, D., and Kamath, H.(2015) Dynamic control and optimization of distributed energy resources in a microgrid. IEEE Trans. Smart Grid, 6:2884-2894.

4. Seidi Khorramabadi, S.,, and Bakhshai, A. (2015) Critic-based self-tuning PI structure for active and reactive power control of VSCs in microgrid systems. IEEE Trans. Smart Grid, 6:92-103.

5. Li, X., Li, Y., and Seem, J. E. (2013) Maximum power point tracking for photovoltaic system using adaptive extremum seeking control. IEEE Trans. Control Syst. Tech., 21:2315-2322. 